\title{
The effects of temporal waveform upon apparent contrast
}

\author{
FREDERICK L. KITTERLE \\ University of Toledo, Toledo, Ohio \\ and \\ THOMAS R. CORWIN \\ Center for Visual Science, University of Rochester, New York
}

\begin{abstract}
Temporal contrast enhancement (TCE) refers to the finding that the apparent contrast of flashed low-spatial-frequency gratings is higher for flashes of intermediate duration (i.e., 50$120 \mathrm{msec}$ ) than for flashes of longer duration. The present experiment investigated the effect of temporal waveform upon TCE. We measured the apparent contrast of a .6-cycles/deg sinusoidal grating that varied in duration. Four temporal waveforms were used: abrupt onset/ abrupt offset, abrupt onset/gradual offset, gradual onset/abrupt offset, and gradual onset/ gradual offset. TCE was found only for targets with abrupt onsets, regardiess of offset waveform. These results have implications about the role of onset transients in the coding of brightness.
\end{abstract}

The brightness of a visual stimulus is not determined solely by its luminance but also depends on its size, color, contrast, and duration. Stimulus duration, in particular, affects brightness in a complex way: under some conditions, intermediate-duration flashes (i.e., $50-100 \mathrm{msec}$ ) often appear brighter than long-duration flashes of the same luminance. This effect has been called the Broca-Sulzer phenomenon. For convenience, we will refer to the effect here as temporal brightness enhancement (TBE).

Theorists have attempted to account for TBE by identifying some aspect (of the visual response to a flash) that varies in a nonmonotonic way with respect to flash duration. They then assume that this aspect mediates flash brightness. One theory suggests that brightness is mediated by the neural response to flash onset (Adrian, 1928; Boynton \& Kandel, 1957). Conversely, other theorists have suggested that neural off-responses are more highly correlated with perceived brightness. For example, Kelly and Savoie (1978) have suggested that the magnitude of the neural "undershoot" associated with flash offset varies nonmonotonically with flash duration. Such off-responses might therefore mediate TBE.

Indirect evidence about the role of transients in brightness enhancement was obtained by Kitterle and Corwin (1979). They measured the influence of spatial frequency upon changes in the apparent con-

F. L. Kitterle's mailing address is: Department of Psychology, University of Toledo, Toledo, Ohio 43606. T. R. Corwin's mailing address is: Center for Visual Science, University of Rochester, Rochester, New York 14627. trast of a grating as a function of duration. They showed a temporal contrast-enhancement effect (TCE) for low- but not high-spatial-frequency gratings. Other psychophysical studies have shown that flashed low-spatial-frequency gratings produce transient threshold elevations at onset and of fset, whereas high-spatial-frequency flashes do not (Kelly, 1971; Kelly \& Savoie, 1978; King-Smith \& Kulikowski, 1975). Kitterle, Corwin, and Berta (1979) and Mitov, Vassilev, and Manahilov (1981) have confirmed this result with suprathreshold gratings in a masking paradigm.

The magnitude of the initial transient response to a flash can be reduced by "ramping" its onset (i.e., turning it on gradually) (Matsumura, 1976; Tolhurst, 1975). Thus, if transient activity plays a role in TBE and TCE, then ramped flashes should show little or no enhancement. In order to compare the relative importance of onset and offset transients, we presented stimuli with four temporal waveforms: abrupt onset/abrupt offset, abrupt onset/gradual offset, gradual onset/abrupt offset, and gradual onset/gradual offset. Two observers judged the apparent contrast of gratings presented at each waveform as a function of flash duration.

\section{METHOD}

\section{Observers}

Two experienced psychophysical observers, F.L.K. (39 years old) and T.R.C. (44 years old), participated in this experiment. F.L.K. had normal vision; T.R.C. had corrected-to-normal vision.

\footnotetext{
Apparatus

The gratings were generated on the face of a Tektronix 608 monitor; the screen was masked down so that it subtended a
} 
visual angle of $7.3 \mathrm{deg}$ (width) $\times 5.6 \mathrm{deg}$ (height) at a viewing distance of $104.1 \mathrm{~cm}$. Throughout the experiment, gratings of $.61 \mathrm{cycles} / \mathrm{deg}$ and $34 \%$ contrast (when turned on and off abruptly) were used. In the ramped conditions, contrast change was adjusted so that all flashes at a given duration had the same total interrated energy. Duration for the ramped conditions was defined as the time between the half-amplitude points. A photodiode and auxiliary oscilloscope were used to calibrate the temporal characteristics of the gratings. The frame rate was set at $20 \mathrm{kHz}$. A microprocessor (Ohio Scientific C4P) provided voltage values to a voltage-controlled amplitude generator that was used to vary the onset and/or offset of the grating. In the ramped conditions, either the onset or the offset was a $20-\mathrm{msec}$ ramp. The mean luminance of the screen was $2.01 \mathrm{fL}$. The display was viewed binocularly.

\section{Procedure}

At the beginning of the experimental session, the observer adapted to the mean luminance of the display for $3 \mathrm{~min}$. Then the grating target was presented successively to the same retinal region twice, with a delay of $1.5 \mathrm{sec}$ between presentations. It was determined empirically that this duration was sufficiently long to ensure that the two pulses did not interact. A given trial consisted of the presentation of two flashes, one exposed for a duration of $\mathrm{T}$ msec and the other for a duration of $2 \mathrm{~T}$ msec. The order in which each was presented, as well as the value of each, was varied randomly over trials. There was a 2-sec intertrial interval, during which the observer was required to press one of two response keys to indicate which of the two flashes had the greater contrast.

The format used to display these data is similar to that of Bowen and Pokorny (1978). In this format, the percentage of trials on which the longer flash is judged to have more contrast is plotted as a function of the shorter flash duration. For short-duration flashes (for which apparent contrast increases monotonically with duration), the longer flash should always be judged to have more contrast than the shorter flash. If TCE occurs, then there will be some flash duration $T$ for which a flash of duration $2 \mathrm{~T}$ will never be judged higher in contrast. Finally, in the range in which contrast is independent of duration, the longer flash should be judged higher in contrast than the shorter flash in approximately $50 \%$ of the trials.

\section{RESULTS}

The results are plotted in Figure 1A for F.L.K. and Figure $1 B$ for T.R.C. These figures show the percentage of time the longer flash was judged to have more contrast than the shorter flash. In these figures, the four temporal waveforms are plotted as follows: abrupt onset/abrupt offset (filled circles), abrupt onset/gradual offset (filled triangles), gradual onset/abrupt offset (open triangles), and gradual onset/gradual offset (open circles).

Since the results of both observers are essentially the same, this discussion focuses upon the results of F.L.K. Note that filled data points (circles and triangles) dip significantly below $50 \%$ at intermediate durations. The data plotted with the open circles and open triangles do not show such a drop. This indicates that flashes with abrupt temporal onset yield TCE, whereas the gradual turning on of the flashes abolishes TCE. It is also quite evident in these data that target offset does not play a significant role in temporal contrast enhancement.
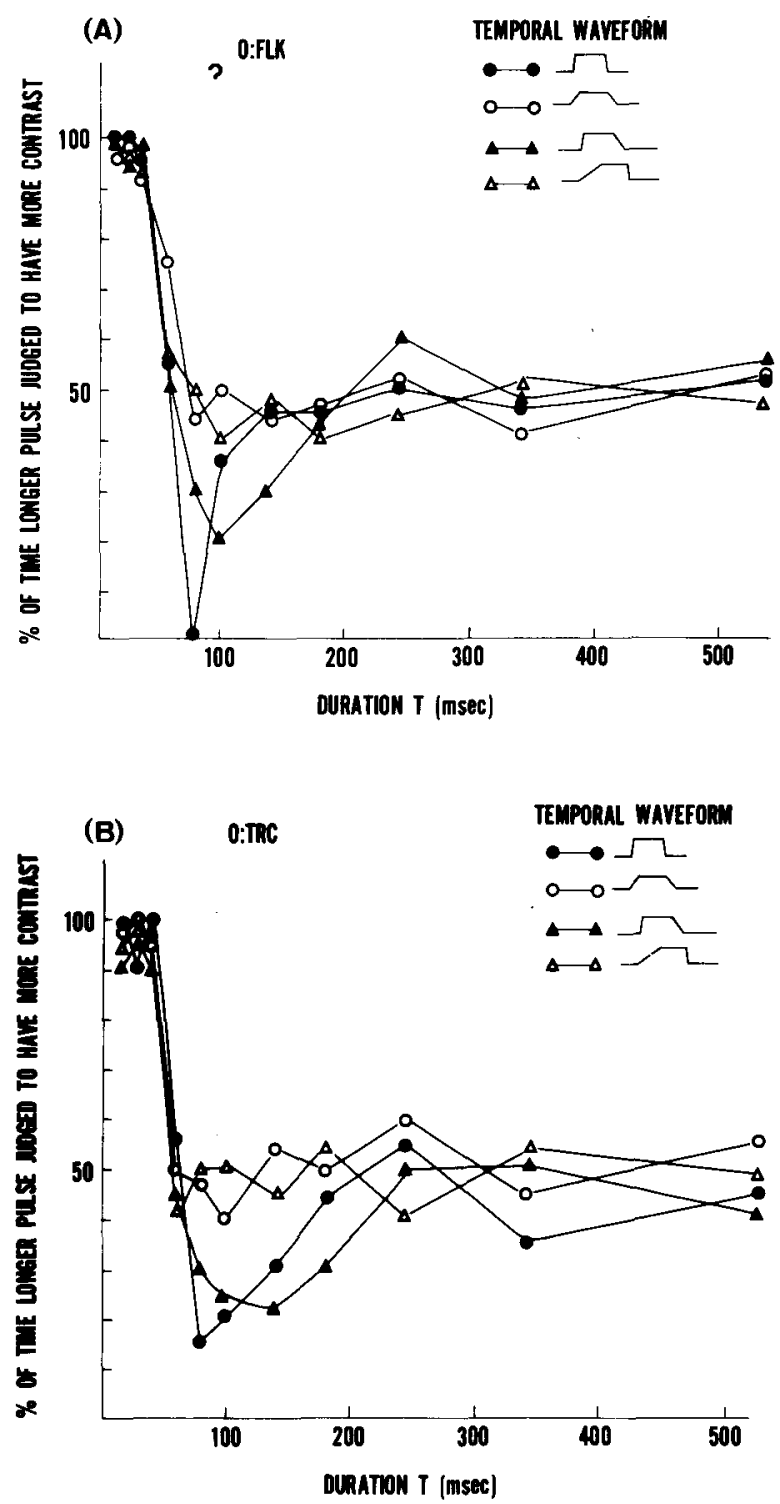

Figure 1. (A) Results for Observer F.L.K. (O:FLK), showing the percentage of trials that the longer (2T) flash was judged to have the greater contrast than the shorter ( $T$ ) fash. The parameter of these figures is the temporal waveform, which varies as follows: abrupt onset/abrupt offset (filled circles), abrupt onset/gradual offset (filled triangles), gradual onset/ abrupt offset (unfilled triangles), and gradual onset/gradual offset (unfilled clrcles). Each data point represents 60 judgments/condition. (B) Corresponding results for Observer T.R.C. (O:TRC). Each data point represents 30 judgments/condition.

\section{DISCUSSION}

The results of the present experiment indicate that response transients associated with flash onset are important in the coding of contrast. For flashes with ramped onsets, TCE is abolished; that is, intermediate-duration flashes have the same apparent contrast as long-duration flashes. Conversely, 
offset transients do not appear to influence TCE. Although gradual offset reduces the magnitude of the offset transient (Matsumura, 1976), flashes having an abrupt onset and gradual offset produced curves similar to those obtained for flashes having both abrupt onset and abrupt offset. Although offset transients do not mediate TCE under the present conditions, they may be involved in the coding of apparent contrast. For example, offset transients may mediate the contrast or brightness of longduration flashes, so that when two long-duration flashes of similar duration are compared, a flash with a ramped offset appears to be dimmer or to have less contrast than one with an abrupt offset. This hypothesis is supported by Barlow and Verrillo (1976), who found no difference in the brightness of two long-duration flashes of equal luminance, one with ramped onset and the other with abrupt onset. Evidently on-response information was not utilized to code brightness in this case.

Although we cannot yet specify how a neural response to a flash of a given duration is coded into a contrast or brightness response, the present results do show that transients play a role in coding the perceived contrast of intermediate duration flashes. There has been some suggestion that the brightness of long flashes is based upon neural activity in the later portions of the flash, whereas the brightness of short- and intermediate-duration flashes is based upon the onset transient (Broca \& Sulzer, 1902; McDougall, 1904). This hypothesis implies a criterion shift in coding flash brightness as duration varies. It is possible, furthermore, that some observers may utilize onset transients to code brightness and that other observers may not. Recent research suggests substantial individual differences in the coding of brightness (Bowen \& Markell, 1980; Bowen, Sekuler, Owsley, \& Markell, 1982).

On the basis of these facts and of the results of this study, we conclude that neural on-response activity is a necessary, but not a sufficient, condition for obtaining TBE or TCE.

\section{REFERENCES}

Adnine, E. D. The physical basis of sensation: The action of sense organs. London: Christophors, 1928.

BarLow, R. B., \& Verrillo, R. Brightness sensation in a ganzfeld. Vision Research, 1976, 16, 1291-1298.

Bowen, R. W. \& MarKeLl, K. A. Temporal brightness enhancement studied with a large sample of observers: Evidence for individual differences in brightness perception. Perception \& Psychophysics, 1980, 27, $465-476$.

Bowen, R. W., \& Poxorny, J. Target edge sharpness and temporal brightness enhancement. Vision Research, 1978, 18, 1691-1695.

Bowen, R. W., Sexuler, R., Owsley, C. J., \& Markell, K. A. Individual differences in pulse brightness perception. Perception \& Psychophysics, 1982, 30, 587-593.

Boynton, R. M., \& KandzL, G. On responses in the human visual system as a function of adaptation level. Journal of the Optical Society of America, 1957, 47, 275-286:

Broca, D., \& Sulezen, A. La sensation luminise en fonction du temps. Journal Physiologie, 1902, 4, 632-640.

KELLY, D. H. Theory of flicker and transient responses. I. Uniform fields. Journal of the Optical Society of America, 1971, 61, 537-546.

Kelly, D. H., \& SAvore, R. E. Theory of flicker and transient responses. III. An essential non-linearity. Journal of the Optical Society of America, 1978, 68, 1481-1490.

Kino-Smith, P. E., \& Kulixowski, J. J. Pattern and flicker detection analyzed by subthreshold summation. Journal of Physiology, 1975, 249, 519-548.

Kitrenes, F. L., \& Conwin, T. R. Enhancement of contrast in flashed sinusoidal gratings. Vision Research, 1979, 19, 33-39.

Kitterle, F. L., Conwin, T. R., \& Berta, J. Masking of sinusoidal targets by uniform fields of unequal duration. Journal of the Optical Society of America, 1979, 69, 1445.

Matrumura, M. Visual masking by luminance increment and decrement: Effects of rise time and decay time. Tohoku Psychologia Folia, 1976, 35, 104-114.

McDouanll, $W$. The variation of the intensity of visual sensation with the duration of the stimulus. British Journal of Psychology, 1904, 1, 151-189.

Mitov, D., Vaballev, A., \& Manahilov, V. Transient and sustained masking. Perception \& Psychophysics, 1981, 30, 205-210.

ToLHurer, D. J. Sustained and transient channels in human vision. Vision Research, 1975, 15, 1151-115s.

(Manuscript received July 20, 1982; revision accepted for publication October 22, 1982.) 